\title{
Surface and Coatings Technology
}

Volume 204, Issue 15, 25 April 2010, Pages 2483-2486

One-step fabrication process of superhydrophobic green coatings

D.K. Sarkar,

N. Saleema

Centre Universitaire de Recherche sur l'Aluminium (CURAL), L'Université du Québec à Chicoutimi, 555 Boulevard de l'Université, Chicoutimi, Québec, Canada G7H 2B1 Received 14 September 2009, Accepted 21 January 2010, Available online 1 February 2010

doi:10.1016/j.surfcoat.2010.01.033

\section{Abstract}

In general, creation of superhydrophobic surfaces is composed of two steps: (i) creation of a rough surface and (ii) passivation of the surface with the low surface energy molecules or coatings. Superhydrophobic properties cannot be achieved on a surface without these two essential factors fulfilled. In the present work we have demonstrated that superhydrophobic silver films on copper $(\mathrm{Cu})$ substrates can be created in just a one-step process via galvanic reactions by immersing the Cu substrates in silver nitrate solution containing benzoic acid, simplifying the complexity of two different steps involved in the former method. Silver films were also fabricated using similar process without benzoic acid for comparative studies. The X-ray diffraction (XRD) and Fourier transform infrared spectroscopy (FTIR) confirmed the formation of benzoic acid incorporated silver films. Scanning electron microscopy (SEM) images showed micronano structured leaf-like and flower-like morphological features in the films prepared without and with benzoic acid, respectively. Benzoic acid incorporated flower-like silver films demonstrated water repellency as the water drops rolled off those surfaces whereas complete absorption of water drops were encountered on the leaf-like silver surfaces prepared without benzoic acid.

Keywords : Silver; Electroless deposition; Scanning electron microscopy (SEM); X-ray diffraction; Nanostructure; Superhydrophobic green coatings 


\section{Introduction}

Technology is frequently the application of science, which is in turn inspired by nature. The technology used by many researchers in making superhydrophobic surfaces is one such example. Numerous plant surfaces and body parts of certain insects and animals exhibit superhydrophobic properties, evolved to survive many of nature's hazards by repelling dirt and dust particles in rain [1], [2] and [3]. Due to the tremendous importance of superhydrophobic surfaces in today's emerging technologies, many efforts have been made to replicate nature. The term "bio-mimicking" is commonly used to denote the artificial production of superhydrophobic surfaces as the technology to make them involves copying nature's solutions. The applications of superhydrophobic surfaces are diverse due to their unique water-repellency and self-cleaning abilities. The most common areas where superhydrophobic surfaces attract attention include anti-biofouling paints for boats [4], bio-chips [5], biomedical applications [6], microfluidics [7], corrosion resistance [8], eyeglasses, self-cleaning windshields for automobiles [9], stain resistant textiles [10], anti-sticking of snow for antennas and windows [11] and many others. In a recent review, we have demonstrated that superhydrophobic nanostructured surfaces may play an important role on anti-icing effects which may have diverse applications as coating on electric cables and insulators, aircraft wings, ship hulls, glass structures, windshields, etc. [12]. A recent book entitled "Superhydrophobic Surfaces" edited by K. L. Mittal and A. Carré has also illustrated several preparation techniques and the various applications of superhydrophobic surfaces [13].

The approaches used in making a surface superhydrophobic emphasize the importance of a combination of micro-nanoroughness on the surface and low surface energy as nature teaches us. Mostly, the technique used involves a two-step procedure in which a surface is first roughened and then modified to lower the surface energy [14], [15] and [16]. Creation of certain micro-nanoroughness as a first step promotes the entrapment of air in the spacings between the rough features; and lowering the surface energy as a second subsequent step helps reduce the affinity of water drops with the low energy surface thereby weakening the water-surface interaction. We have, in the last two years fabricated several superhydrophobic surfaces using diverse methods, via two-step processes, which demonstrated water rolling-off properties [12], [17], [18], [19], [20] and [21]. In those works, methods such as chemical bath deposition (CBD), substrate chemical etching, galvanic exchange reaction, etc., have been carried out in obtaining the surface roughness and passivation methods such as immersion in organic 
acid such as stearic acid, fluoroalkyl silane molecules or by coating with rf-sputtered Teflon have been carried out in obtaining low energy surfaces. However, the complexity in the conventional practice of involving two steps, each step involving multiple procedures in certain methods of preparing superhydrophobic surfaces can be simplified if the same could be fabricated in just one step. Incorporation of low surface energy ingredients such as organic acids during the process of surface roughness creation may lead to obtaining superhydrophobic surfaces in just one step. Brankovic et al. have previously reported incorporation of saccharin molecules in CoFe alloys with a view to improving the corrosion resistance properties of these alloys [22].

Organic acids such as benzoic acid and their salts are known to inhibit the growth of mold, yeast and some bacteria and are also used as food preservatives [23] and [24]. Similarly, metals such as silver, which can be easily deposited, for example on copper surfaces, via galvanic reactions [18] or phenomenon also known as cementation processes [25] and [26], are used as an antibacterial material in bio-medicine [27] and [28]. Therefore, use of such biocompatible materials as ingredients in making superhydrophobic surfaces will provide a new pathway to making antibacterial, non-toxic and environmentally friendly superhydrophobic surfaces for which reason; such surfaces might be termed "superhydrophobic green coating". The term "green" here mainly refers to non-toxicity, environment friendly and biocompatibility. Such a green coating can have a tremendous potential in applications where environment and energy is concerned. For example, superhydrophobic green coatings, if coated in the interior of drinking water pipes can inhibit corrosion and reduce drag eliminating health risks as well as aiding in reducing energy consumption.

In this article, we report the fabrication of superhydrophobic green silver coating on copper substrates in a one-step wet chemical process by incorporating benzoic acid in the silver film during their deposition owing to their biocompatible and environmental friendly properties.

\section{Experiment}

Thin silver films were coated at room temperature $\left(22^{\circ} \mathrm{C}\right)$ on clean copper substrate surfaces by simply immersing them for a certain period of time ( $1 \mathrm{~min}$ to $1080 \mathrm{~min}$ ) in aqueous silver nitrate solution $(1 \times 10-3 \mathrm{M}$ to $100 \times 10-3 \mathrm{M})$ with or without the presence of desired concentration of benzoic acid. The X-ray diffraction (XRD) analysis of the silver films coated surfaces was carried out using Bruker D8 Discover system. The 
morphological and elemental analyses were performed using JEOL (JSM 6480 LV) scanning electron microscope (SEM/EDX). The wetting characteristics of the samples surfaces were carried out using a First Ten Angstrom contact angle goniometer. Atomic bonding of the benzoate formation was characterized using Fourier transform infrared spectroscopy (FTIR) (Perkin-Elmer Spectrum One).

3. Results and discussion

Fig. 1 shows the X-ray diffraction (XRD) pattern of the (a) copper substrates, (b) the silver films deposited on the copper substrates by galvanic reaction in silver nitrate solution and (c) the silver films deposited on the copper substrates using silver nitrate solution in the presence of benzoic acid molecules. In the $2 \theta$ scan range of $35-85^{\circ}$ (

Fig. 1(a)) copper substrates show characteristic peaks of $\mathrm{Cu}(111), \mathrm{Cu}(200)$ and $\mathrm{Cu}(220)$ at $43.4^{\circ}, 50.53^{\circ}$ and $74.16^{\circ}$, respectively, which are in good agreement with the crystallographic data [29]. The silver films deposited by galvanic ion exchange reaction ( Fig. 1(b)) show the characteristic peaks of $\mathrm{Ag}$ (111), $\mathrm{Ag}(200), \mathrm{Ag}(220), \mathrm{Ag}(311)$ and $\mathrm{Ag}(222)$ at $38.18^{\circ}, 44.38^{\circ}, 64.53^{\circ}, 77.48^{\circ}$ and $81.65^{\circ}$, respectively, confirming the silver deposition [30]. The origin of the three peaks at $36.5^{\circ}, 42.35^{\circ}$ and $61.43^{\circ}$ are due to the formation of copper oxide (Cu2O) crystalline phase, which is also complemented by the black colored appearance of the deposited films [31]. However, it has been observed that in silver films deposited with the presence of benzoic acid molecules in the silver nitrate solution ( Fig. 1(c)), the three peaks of $\mathrm{Cu} 2 \mathrm{O}$ are completely disappeared. These films appeared to be pure silver as evident from the distinct peaks of $\mathrm{Ag}(111), \mathrm{Ag}(200)$, $\mathrm{Ag}(220)$, and $\mathrm{Ag}(311)$ at $38.18^{\circ}, 44.38^{\circ}, 64.53^{\circ}$, and $77.48^{\circ}$, respectively [30]. However, in the present work, addition of benzoic acid with the silver nitrate solution resulted in pure silver films without any trace of copper oxide in the films. Such films would have potential application in the silver metallization technologies at a low cost [32] and [33]. 


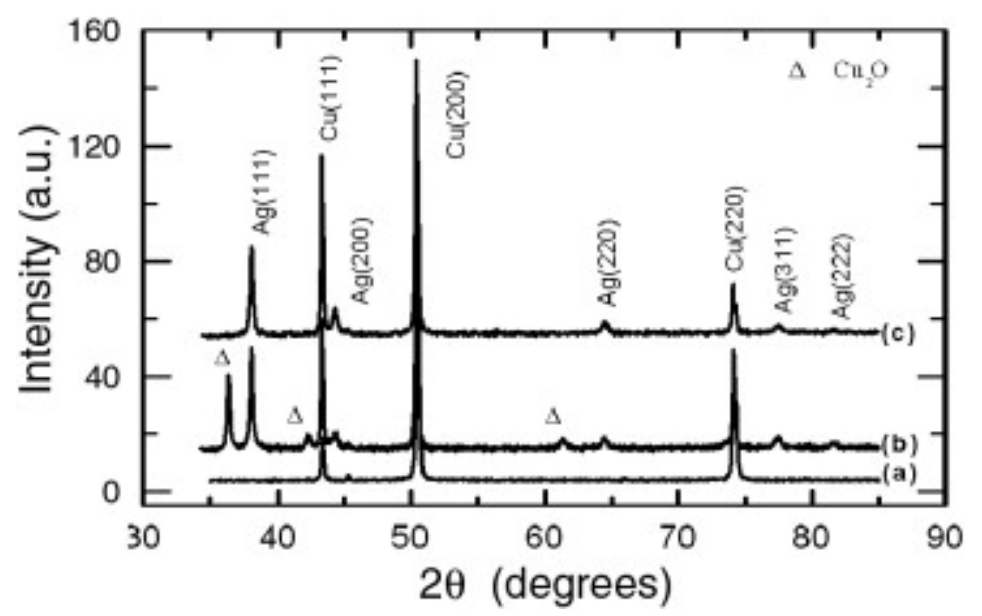

Fig. 1.

XRD patterns of (a) copper substrate, (b) the silver film deposited on galvanic exchange reaction on copper substrate in the silver nitrate solution, and (c) the silver film deposited on galvanic exchange reaction on copper substrate in the silver nitrate solution with benzoic acid molecules.

Fig. 2 shows the XRD pattern of the silver powder collected from the films deposited on copper substrates in presence of benzoic acid in the silver nitrate solution for a deposition time of $60 \mathrm{~min}$. The diffraction study of the powder was carried out keeping the powder on a single crystal $\mathrm{Si}(100)$ substrate. The diffraction study of the powder was carried out up to $68^{\circ}$ as $\mathrm{Si}(400)$ usually gives a very strong peak at $69.19^{\circ}$ from the $\mathrm{Si}(100)$ single crystal substrates that might damage the detector. Three distinct peaks of $\mathrm{Ag}(111), \mathrm{Ag}(200)$, and $\mathrm{Ag}(220)$ have been observed in the range of $35^{\circ}$ to $68^{\circ}$. The sharp peak at $61.71^{\circ}$ is due to the diffraction peak from $\mathrm{Si}(400)$ of $\mathrm{Cu} K \beta$ lines. The inset shows the low angle XRD pattern in the $2 \theta$ range of $6-18^{\circ}$ of the same powder showing three peaks from copper-3-hydroxy benzoate [34]. However, in the silver thin films of Fig. 1(c), we did not observe any peaks in the low angle. 


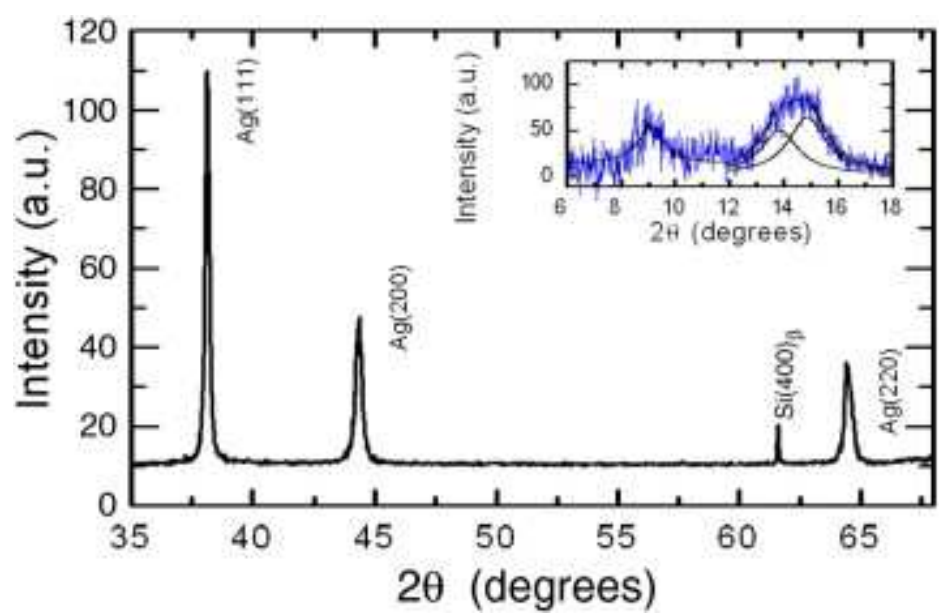

Fig. 2. XRD pattern of silver powder collected from the films deposited in the presence of benzoic acid. Inset shows the XRD pattern of the same in low angle.

The morphological features of the two films prepared with or without benzoic acids are found to vary to a great extent (Fig. 3) The leaf-like surface (fractal structure) morphology (Fig. 3(a)) of the films (composed of micro-nano patterned) prepared without benzoic acid which is in resemblance to our previously reported structures [18]. However, the morphological features changed to flower-like structure (composed of micro-nano patterned) as shown in Fig. 3(b) when benzoic acid was added in the silver nitrate solution. The inset of Fig. 3(b) shows a water droplet ( $8 \mu \mathrm{L})$ on those flower-like films prepared in the presence of benzoic acid. The water contact angle (CA) and contact angle hysteresis obtained on these films is $\sim 162^{\circ}$ and $\sim 2^{\circ}$ respectively. The water drops were found to roll off those surfaces easily even with slightest tilt of the surface. The high water contact angle and water repellency demonstrated by the rolling water drops is attributed to the combination of the rough flower-like morphological features of this film and the low surface energy of benzoic acid incorporated into these films simultaneously during their growth in the galvanic exchange reaction with copper substrates. The low surface energy of benzoic acid arises from the presence of the low surface energy $-\mathrm{CH}$ groups in the benzoic acid. These films, for their superhydrophobic properties obtained using biocompatible ingredients might be termed "superhydrophobic green silver coating". We have previously reported the rolling off of water drops on fractal-structured silver surfaces by two-step processes using stearic acid molecules for passivation [18]. Also, Gu et al., have recently reported extreme-hydrophobicity of $\mathrm{Ag}$ on Cu surfaces by galvanic exchange reactions [35]. On the other hand, we have observed that water drops just absorbed completely in the leaf-like structure ( Fig. 3(a)) prepared 
without benzoic acid making it impossible to measure the water contact angle on its surface. This behavior can be accounted to the highly microporous nature of the fractalstructured high surface energy silver films. Therefore, no inset of water drop image was possible to provide for this leaf-like fractal surface. Following our recent work [18], Gu et al. [35] have very recently studied the morphology and wetting characteristics of silver films deposited on Cu via galvanic reactions based on the changes in the concentration of silver nitrate solution and their deposition time. They found that the as-deposited silver films grown using $1 \mathrm{mM} \mathrm{AgNO} 3$ solution for a duration of $45 \mathrm{~min}$ provided a CA of only $35^{\circ}$ and the film produced using $10 \mathrm{mM} \mathrm{AgNO3}$ solution for 25 min provided a CA of only $10^{\circ}$. Their observation demonstrates the dependence of CA on the difference in concentration influenced morphological features, with silver films prepared using $10 \mathrm{mM}$ AgNO3 showing comparably highly microporous fractal like structure than the nanoporous film prepared using $1 \mathrm{mM}$ AgNO3 solution. In our case, we observed a compete absorption of the water drop on the film produced using much higher concentration than reported by Gu et al. [35] which would obviously be expected to have much higher micro-porosity as shown in SEM image of Fig. 3(a). However, in presence of benzoic acid, the morphological feature of the films change to flower-like structure from a leaf-like morphology on those without benzoic acid ( Fig. 3(b)). This change can be attributed to the rate of reaction influenced by the presence of benzoic acid in the solution. Further work is currently in progress to understand the growth mechanism (both crystallographic and morphological) of the silver film influenced by the presence of benzoic acid. Apart from creation of microporous silver films by galvanic exchange reactions [18] and [35], Viyannalage et al. have shown that the nanoporous morphological feature of the deposited silver can be controlled also by applied potential in the electrochemical deposition process [25]. Also, Sarkar et al. have earlier shown that the morphological change of electrochemically deposited copper films on conducting polymer can be controlled from highly monodispersive nanoparticles to the micrometer size fractal structures by applied potential [36]. 

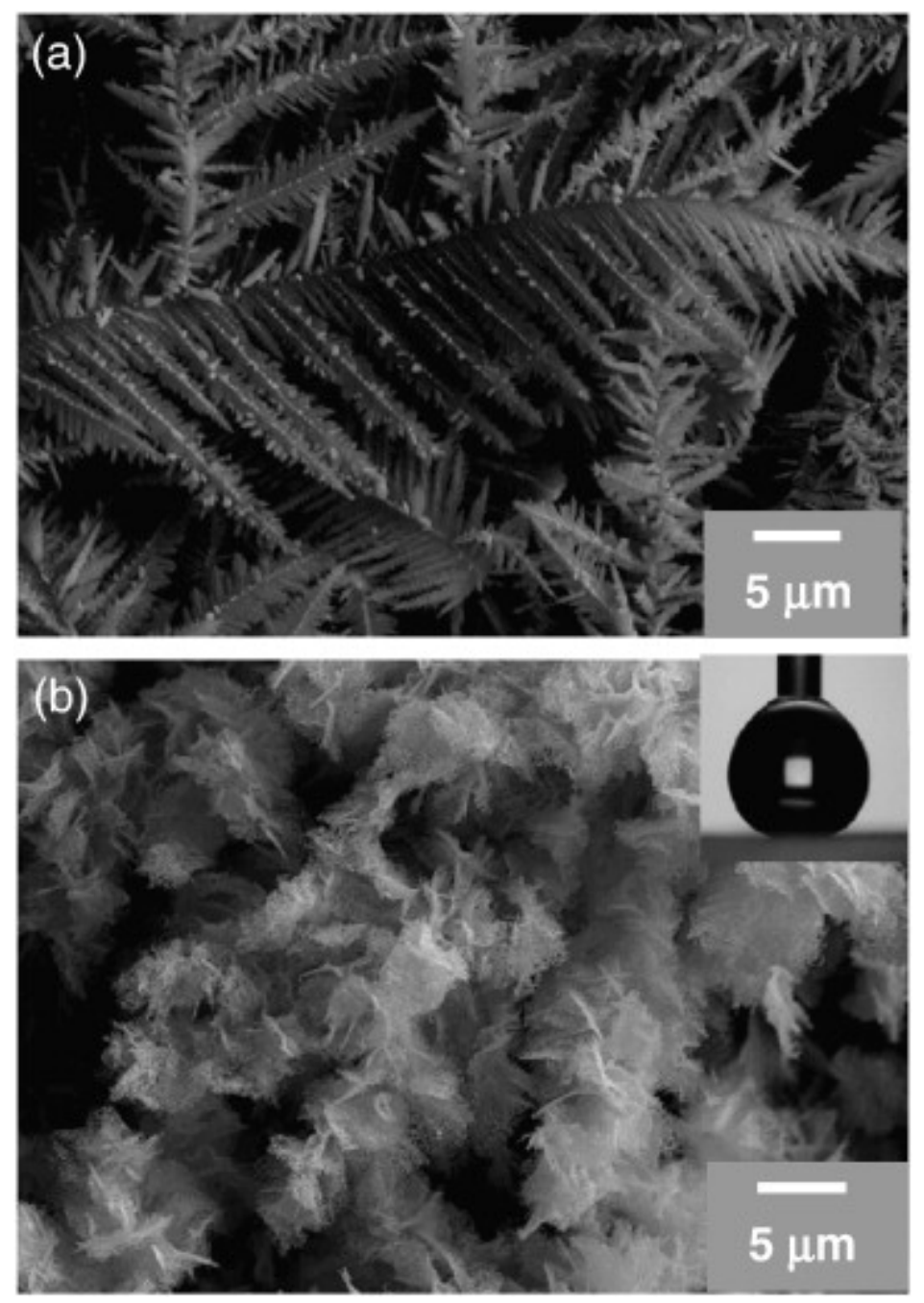

Fig. 3. SEM images of silver films deposited on copper substrates by galvanic exchange reaction in the silver nitrate solution: (a) without benzoic acid and (b) with benzoic acid. Inset shows the drop of water on the silver surface prepared with benzoic acid molecules.

Fig. 4 shows the energy dispersive $X$-ray analysis (EDX) spectra of the silver films deposited on copper substrates (a) in silver nitrate solution and (b) in silver nitrate solution with benzoic acid molecules. Fig. 4(a) shows the distinct peaks of silver (2.98 keV), copper (0.92 keV) and oxygen (0.53 keV) in the films. The presence of oxygen in those films with the peak at $0.53 \mathrm{keV}$ in the EDX spectrum is complemented with the observation of $\mathrm{Cu} 2 \mathrm{O}$ by XRD as shown in Fig. 1(b). EDX spectrum of silver films prepared with the benzoic acid (Fig. 4(b)) shows the presence of two distinct elements, namely, copper $(0.92 \mathrm{keV})$ and silver $(2.98 \mathrm{keV})$ with a trace of carbon with the peak at 
$0.28 \mathrm{keV}$. The absence of oxygen in this spectrum (Fig. 4(b)) is well complemented with the XRD analysis (Fig. 1(c)) that confirmed the deposition of $\mathrm{Cu} 2 \mathrm{O}$ free pure silver films. The presence of carbon in this EDX spectrum confirms the incorporation of benzoic acid molecule in these films. The bonding characteristics of the carbon in these films have been further characterized by FTIR and are shown in the inset of Fig. 4(b). The distinct peak at $1386 \mathrm{~cm}-1$ is due to the presence of COO- vibration bond with copper possibly resulting in the formation of copper-3-hydroxy benzoate $(\mathrm{C} 7 \mathrm{H} 5 \mathrm{CuO} 3)$ complementing the XRD analyses. The adsorption of benzoic acid on copper surfaces shows that the COO- vibration bond varies from 1390 to $1405 \mathrm{~cm}-1$ depending on the orientation of the benzoic acid with respect to the surface [37].

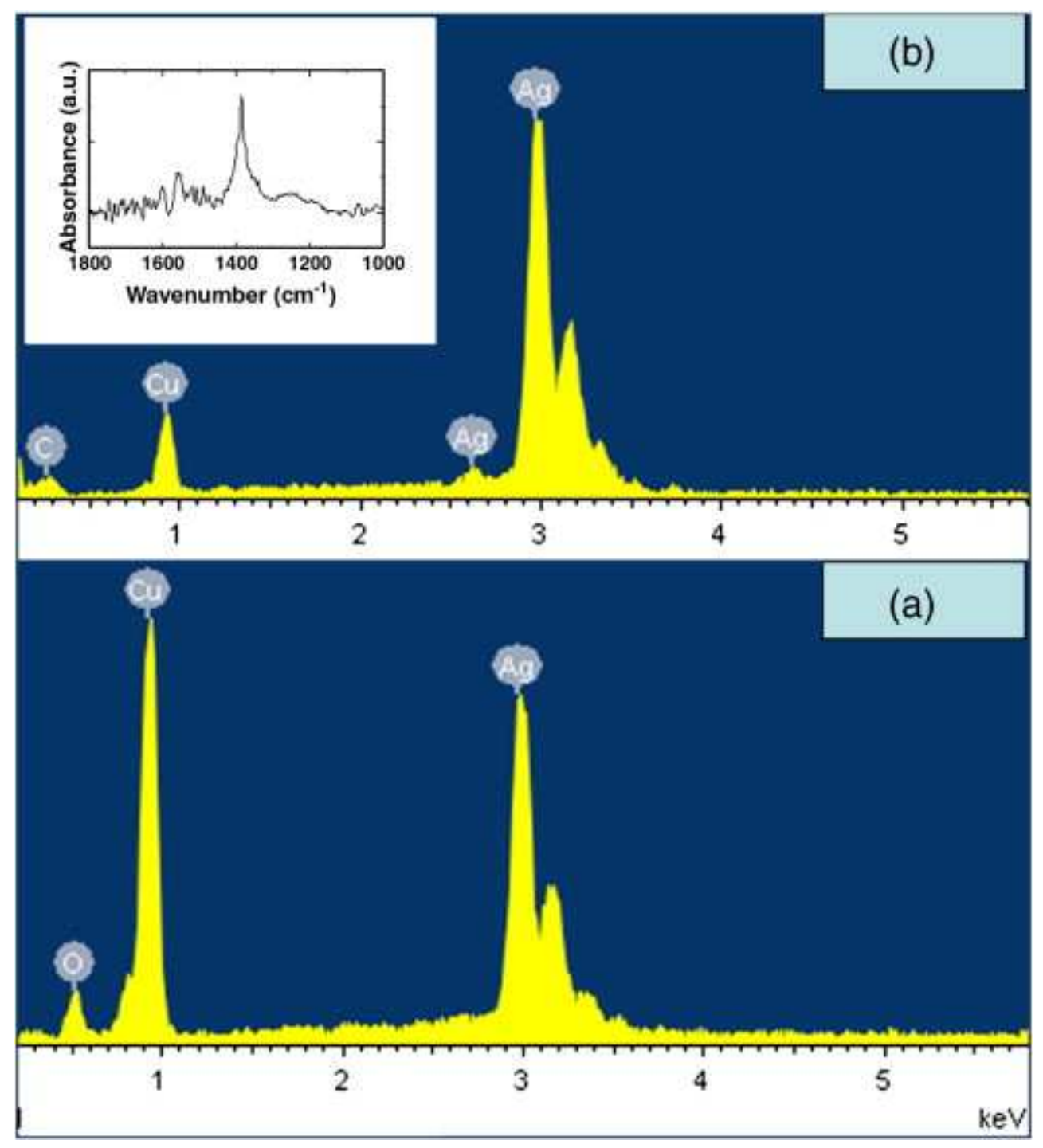


Fig. 4. EDX spectra of silver films prepared in (a) silver nitrate solution and (b) silver nitrate solution with benzoic acid molecules. Inset shows the FTIR spectra of silver powder collected from the silver films prepared with the benzoic acid molecules.

We report that superhydrophobic green silver coating on copper surface is possible by galvanic exchange reaction due to the feeble electrochemical potential difference between $\mathrm{Ag}+$ ions and copper. However, this process can be generalized for any substrates, either conductor, semiconductor or insulator by activating their surfaces with SnCl2 prior to electroless silver coatings [38]. Due to the possibility of making superhydrophobic green silver coatings on any substrates, such films can have tremendous potential applications in the fields of antibacterial, anti-biofouling, anticorrosion, drag reduction and energy conservation, microfluidics, self-cleaning, etc.

\section{Conclusion}

In conclusion, we have developed a novel one-step process to fabricate benzoic acid incorporated superhydrophobic silver thin films on copper substrates. The water contact angle on these films is found to be $\sim 162^{\circ}$ with the rolling-off properties of water drops. Owing to the antibacterial and environmental friendly properties these coatings have been called "superhydrophobic green coatings". These superhydrophobic green silver coatings might find its potential use where non-toxicity, friendly environment, health and energy are of concern.

\section{Acknowledgements}

DKS would like to acknowledge the Dean of Graduate studies, UQAC, Ms. N. Bouchard for providing a start-up funding as well as Prof. D. Marceau, the Director of CURAL for financial support in purchasing the necessary chemicals for this research work. DKS also likes to thanks Lory Brisson, and Mathieu Brisson for their assistance and Caroline Potvin for providing the benzoic acid and the FTIR facility. DKS also likes to thank Prof. Kocaefe for providing the facility to measure the water contact angle.

\section{References}

[1] W. Barthlott, C. Neinhuis

Planta, 202 (1997), p. 1 
[2] X. Gao, L. Jiang

Nature, 432 (2004), p. 36

[3] Y. Zheng, X. Gao, L. Jiang

Soft Matter, 3 (2007), p. 178

[4] A. Scardino, R. De Nys, O. Ison, W. O'Connor, P. Steinberg

Biofouling, 19 (2003), p. 221

[5] J.D.J.S. Samuel, P. Ruther, H.-P. Frerichs, M. Lehmann, O. Paul, J. Ruhe

Sens. Actuators B, 110 (2005), p. 218

[6] A. Singh, L. Steely, H.R. Allcock

Polym. Prepr. (ACS, Div. Polym. Chem.), 46 (2005), p. 599

[7] H. Gau, S. Herminghaus, P. Lenz, R. Lipowsky

Science, 283 (1999), p. 46

[8] T. Liu, Y. Yin, S. Chen, X. Chang, S. Cheng

Electrochim. Acta, 52 (2007), p. 3709

[9] D. Quéré

Rep. Prog. Phys., 68 (2005), p. 2495

[10] K. Satoh, H. Nakazumi

J. Sol-Gel Sci. Technol., 27 (2003), p. 327

[11] T. Kako, A. Nakajima, H. Irie, Z. Kato, K. Uematsu, T. Watanabe, K. Hashimoto

J. Mater. Sci., 39 (2004), p. 547

[12] D.K. Sarkar, M. Farzaneh

J. Adhesion Sci. Technol., 23 (2009), p. 1215

[13] K.L. Mittal, A. Carré (Eds.), Superhydrophobic Surfaces, BRILL (2009)

[14] X. Zhang, F. Shi, J. Niu, Y. Jiang, Z. Wang

J. Mater. Chem., 18 (2008), p. 621

[15] Zhi-Guang Guo, Wei-Min Liu, Bao-Lian Su

Appl. Phys. Lett., 92 (2008), p. 063104

[16] K. Liu, M. Zhang, J. Zhai, J. Wang, L. Jiang

Appl. Phys. Lett., 92 (2008), p. 183103

[17] N. Saleema, M. Farzaneh

Appl. Surf. Sci., 254 (2008), p. 2690

[18] A. Safaee, D.K. Sarkar, M. Farzaneh

Appl. Surf. Sci., 254 (2008), p. 2493

[19] D.K. Sarkar, M. Farzaneh 
Appl. Surf. Sci., 254 (2008), p. 3758

[20] D.K. Sarkar, M. Farzaneh, R.W. Paynter

Mater. Lett., 62 (2008), p. 1226

[21] D.K. Sarkar, M. Farzaneh

,in: K.L. Mittal (Ed.), Contact Angle, Wettability and Adhesion, Vol. 5VSP/Brill, Leiden (2008), p. 271

[22] S.R. Brankovic, R. HaisImaier, N. Vasiljevic

Electrochem. Solid-State Lett., 10 (2007), p. D67

[23] A.D. Warth

Appl. Environ. Microbiol., 57 (1991), p. 3410

[24] I. Pastorova, C.G. de Koster, J.J. Boon

Phytochem. Anal., 8 (1997), p. 63

[25] L.T. Viyannalage, Y. Liu, N. Dimitrov

Langmuir, 24 (2008), p. 8332

[26] G.D. Sulka, M. Jaskula

Hydrometallurgy, 70 (2003), p. 185

[27] D.M. Eby, H.R. Luckarift, G.R. Johnson

ACS Nano, 1 (2009), p. 1553

[28] H. Kong, J. Jang

Biomacromolecules, 9 (2008), p. 2677

[29] JCPDS Cu (01-085-1326).

[30] JCPDS, Ag (03-065-8428).

[31] JCPDS: Cu2O (01-077-0199).

[32] S. Buzzi, M. Galli, M. Agio, J.F. Löffler

Appl. Phys. Lett., 94 (2009), p. 223115

[33] F.-J. Haug, T. Söderström, O. Cubero, V.T. Daudrix, C. Ballif

J. Appl. Phys., 106 (2009), p. 044502

[34] JCPDS: copper benzoate (00-031-1624).

[35] C. Gu, H. Ren, J. Tu, T.Y. Zhang

Langmuir, 25 (2009), p. 12299

[36] D.K. Sarkar, X.J. Zhou, A. Tannous, K.T. Leung

J. Phys. Chem. B, Lett., 107 (2003), p. 2879

[37] Q. Chen, C.C. Perry, B.G. Frederick, P.W. Murray, S. Haq, N.V. Richardson

Surf. Sci., 446 (2000), p. 63 
[38] Y. Zhou, J. Chen, Y. Sun, Z. Wang

Mater. Lett., 62 (2008), p. 666 